\title{
RIFUGIATO IN FAMIGLIA. EFFETTI DI SINERGIA TRA ISTITUZIONE PUBBLICA, PRIVATO SOCIALE E CITTADINANZA: L'ESPERIENZA MILANESE
}

Refugee in the family. Synergy effects between public institution, social private and citizenship: the Milanese experience

\author{
Irene Carrano* \\ Daniela Ghiringhelli** \\ Claudia Tagliabue ${ }^{* * *}$
}

\begin{abstract}
Riassunto. L'articolo descrive un progetto di accoglienza in famiglia di rifugiati politici. La sua peculiarità è il coinvolgimento di quattro attori protagonisti: I'ente locale (Comune di Milano), il Terzo Settore, la società civile e il titolare di protezione internazionale. La presenza del Comune e indirettamente del Ministero dell'Interno, finanziatore del progetto, sancisce il riconoscimento istituzionale. L'esperienza, maturata nei quattro anni di sperimentazione, ha consentito di individuare alcune aree tematiche centrali: anzitutto la consapevolezza che tale esperienza comporta, per ciascuno degli attori coinvolti, la rinuncia alla propria zona di comfort per abitare nuovi spazi di azione. I beneficiari, nelle mura domestiche, sperimentano una riappropriazione della propria autonomia; le famiglie diventano protagoniste di una forma generativa di cittadinanza, promotrici di una cultura differente; infine l'istituzione pubblica, in collaborazione con il Terzo Settore, sostiene una forma differente di cura della polis.
\end{abstract}

Parole Chiave: accoglienza; famiglia; integrazione; rifugiati politici.

Abstract. The article describes the project "Rifugiato in famiglia" promoted by the Municipality of Milan targeted to political refugees aiming to support their integration through hosting in Italian citizens' homes. Its main characteristic is the involvement of four key actors: local authority, Third Sector, civil society and holders of international protection. The presence

1 Psicologa, Psicoterapeuta ad indirizzo psicoanalitico. Membro dell'Internationale des Forums du Champ Lacanien. Milano, Italia. E-mail: i.carrano@farsiprossimo.it. Orcid: 0000-0001-5261-6809.

2 Laureata in Sociologia, lavora da 15 anni nella Cooperativa Farsi Prossimo Onlus. E-mail: d.ghiringhelli@farsiprossimo.it. Orcid: 0000-0001-5925-9023.

3 Dottore di ricerca in Sociologia, Organizzazioni e Culture, membro del gruppo di ricerca Relational Social Work presso I'Università Cattolica del Sacro Cuore di Milano. Milano, Italia. E-mail: c.tagliabue@farsiprossimo.it. Orcid: 0000-0002-0472-9224. 
of the municipality and indirectly of the Ministry of the Interior, which is the financier of the project, guarantees the institutional recognition. The experience, gained during the four years of field test, allowed to identify some main thematic areas: first of all the awareness that this experience entails for each actor involved to new spaces for action. Beneficiaries, within the home, experience a reappropriation of their autonomy; families become actors of a generative form of citizenship, promoters of a different culture; finally, the public institution, in collaboration with the Third Sector, supports a different form of Polis care.

Keywords: reception; family; integration; political refugees.

\section{Introduzione: I'accoglienza in famiglia}

Di fronte alle trasformazioni epocali a cui le nuove generazioni stanno assistendo, I'Europa sembra reagire con un incremento della cultura della paura. In diverse nazioni ciò ha preso la forma di un riproporsi di movimenti nazionalistici che, tornati alla ribalta sulla scena politica, hanno cavalcato un diffuso malessere per dare nuovo vigore alle narrazioni avverse al tema migratorio. In questo clima generale la maggior parte delle persone si sono sentite chiamate ad assumere posizioni radicali, schierandosi da una parte o dall'altra, tutto ciò a discapito di una più autentica riflessione su quanto stia realmente accadendo.

Riguardo questo tema, I'Italia non ha fatto eccezione e - anche qui - i discorsi ricorrenti sulla presunta invasione da parte dei profughi hanno spinto le persone a chiedersi "tu da che parte stai?", come se per affrontare quanto sta accadendo fosse più qualificante prendere una posizione che non comprendere e individuare possibili interventi. Fortunatamente però, a partire dal basso, parte della popolazione è riuscita a superare l'impasse, reagendo ad atteggiamenti discriminatori. Sono nati così movimenti solidaristici e di accoglienza che hanno preso forma, alle volte da azioni molto semplici di piccolo volontariato e sono andati via via crescendo fino al punto di divenire una voce che può interloquire con le istituzioni.

Tra le diverse azioni di spinta all'integrazione, che hanno visto il coinvolgimento della popolazione, l'accoglienza in famiglia di rifugiati risulta essere una tra le esperienze più significative. A questo riguardo verrà di seguito presentato il progetto del Comune di Milano "Rifugiato in Famiglia" come esempio di una buona prassi che ha visto negli ultimi quattro anni collaborare in sinergia ente pubblico, Terzo Settore e cittadinanza. Attraverso una metodologia descrittiva, I'articolo inizialmente presenterà la storia e i dati del progetto. Il secondo paragrafo sarà dedicato alle differenti fasi che caratterizzano le singole accoglienze, dal momento della candidatura di famiglie e beneficiari sino a quello dell'uscita di casa. Verrà poi posta l'attenzione su due dimensioni particolarmente significative: la casa e i legami affettivi, anche 
grazie alle parole di chi ha vissuto l'esperienza in prima persona. Nel paragrafo conclusivo verranno approfonditi punti di forza e criticità di un'esperienza che, pure nella sua complessità, mira a favorire processi non solo di riconquista dell'autonomia, da parte dei beneficiari del progetto, ma anche di una integrazione bidirezionale che apra ad una più ampia cura della Polis.

\subsection{II ruolo delle istituzioni nel progetto}

L'aspetto particolarmente rilevante del diffondersi della pratica di accoglienza in famiglia è che tale esperienza ha portato le istituzioni a riconoscere tale fenomeno come possibile risorsa concreta da affiancare all'accoglienza nei grandi centri o negli appartamenti diffusi sul territorio. L'accoglienza in famiglia è infatti entrata a far parte del sistema istituzionale di accoglienza SPRAR (Sistema di Protezione per Richiedenti Asilo e Rifugiati), oggi SIPROIMI (Sistema di Protezione per Titolari di Protezione Internazionale e Minori Stranieri non Accompagnati) ${ }^{1}$. In particolare, a livello nazionale, lo SPRAR definisce I'accoglienza in famiglia come uno strumento che si inscrive all'interno di un percorso personalizzato, da attivare in accordo con il possibile beneficiario. I vantaggi che vengono riconosciuti a questo tipo di strumento riguardano la possibilità di: "prevenire un rischio di vulnerabilità socio-economica dei titolari di protezione internazionale [...] e facilitarne - più di ogni altro intervento - le dinamiche di inclusione sociale" (Ministero dell'Interno, 2015, p. 32).

In tale contesto alcuni comuni sul territorio italiano hanno quindi deciso di attivare questo tipo di accoglienza nelle locali progettualità SPRAR con la supervisione del Servizio Centrale. Tra i Comuni, c'è anche il Comune di Milano che - da oltre quattro anni - ha avviato il progetto "Rifugiato in Famiglia", affidandolo per l'implementazione all'ente gestore dello SPRAR/SIPROIMI (Farsi Prossimo Onlus Soc. Coop. Soc.).

\footnotetext{
Nel 2001 il Ministero dell'Interno Dipartimento per le libertà civili e l'immigrazione, I'ANCI (Associazione Nazionale dei Comuni Italiani) e I'UNHCR (Alto Commissariato delle Nazioni Unite per i Rifugiati) siglarono un protocollo d'intesa per la realizzazione del PNA - Programma Nazionale Asilo, dando vita al primo sistema pubblico per l'accoglienza di richiedenti asilo e rifugiati, diffuso su tutto il territorio italiano, con il coinvolgimento delle istituzioni centrali e locali. La legge n.189/2002 ha successivamente istituzionalizzato queste misure di accoglienza, costituendo lo SPRAR - Sistema di protezione per richiedenti asilo e rifugiati. Attraverso la stessa legge il Ministero dell'Interno ha istituito la struttura di coordinamento del sistema - il Servizio Centrale di informazione, promozione, consulenza, monitoraggio e supporto tecnico agli enti locali - affidandone ad ANCI la gestione. II D.L. 4 ottobre 2018, n. 113, convertito in Legge il 1 dicembre 2018, n. 132, rinomina il Sistema di protezione per richiedenti asilo, rifugiati e minori stranieri non accompagnati (SPRAR) in SIPROIMI: Sistema di protezione per titolari di protezione internazionale e per i minori stranieri non accompagnati. L'accesso al Sistema oggi è riservato ai titolari di protezione internazionale e ai minori stranieri non accompagnati, oltre a i titolari di permesso di soggiorno per: vittime di violenza o tratta, vittime di violenza domestica, motivi di salute, vittime di sfruttamento lavorativo, calamità, atti di particolare valore civile, ma esclude i richiedenti asilo. II SIPROIMI è costituito dalla rete degli enti locali che per la realizzazione di progetti di accoglienza integrata accedono al Fondo nazionale per le politiche e i servizi dell'asilo.
} 


\subsection{L'accoglienza in famiglia a Milano}

Il progetto ha preso avvio al termine del 2015 attraverso la pubblicazione, da parte del Comune di Milano, di un avviso di manifestazione di interesse finalizzato al censimento e all'istituzione di un elenco di famiglie ${ }^{2}$ presenti nel territorio comunale, disponibili ad accogliere nella propria casa adulti titolari di protezione internazionale ${ }^{3}$ per un'esperienza di convivenza della durata di sei mesi.

Dato il carattere innovativo e sperimentale del progetto, il Comune, in accordo con l'ente gestore, ha stabilito inizialmente di dare avvio ad un massimo di cinque accoglienze. Per accompagnare le esperienze di accoglienza è stata inoltre istituita un'équipe multidisciplinare dedicata al progetto e, per fornire un supporto economico alla famiglia, è stato previsto un rimborso spese di $350 €$ mensili.

Nel corso del 2016 sono stati realizzati i primi due cicli di accoglienze, parallelamentel'entegestore ha cominciato a curare la relazione con associazioni di famiglie, presenti sul territorio milanese, già impegnate in altre esperienze di accoglienza. Ciò ha dato avvio alla collaborazione principalmente con due realtà associative (Famiglie per l'Accoglienza, Mondo Comunità e Famiglia), consentendo così di ampliare il numero di famiglie interessate al progetto. Nel corso del 2017 si sono realizzate quindi accoglienze in collaborazione sia con famiglie candidatesi con il bando comunale sia con famiglie provenienti dalle suddette associazioni. Negli anni a seguire le azioni di promozione, individuazione e selezione di nuove famiglie sono divenute parte integrante del progetto stesso. Per questo motivo, analogamente a quanto già accaduto in altre città italiane, il Comune di Milano in collaborazione con l'ente gestore ha scelto di dotarsi di un portale informatico raggiungibile tramite la rete internet, sul quale i cittadini possono reperire informazioni ed eventualmente candidarsi come famiglie ospitanti (www.rifugiatoinfamiglia.it).

Nel quadriennio 2016-2019 il progetto del Comune di Milano "Rifugiato in Famiglia" ha accolto in totale 27 persone, con una prevalenza

2 I requisiti per la partecipazione erano: la residenza anagrafica e il domicilio nel territorio del Comune di Milano; la disponibilità ad uso esclusivo di una stanza per l'accoglienza del rifugiato; la disponibilità a condividere la quotidianità con persone provenienti da diversi contesti socio-culturali e alla collaborazione con le figure professionali del progetto; a titolo preferenziale, ma non esclusivo, l'appartenenza a una rete di famiglie accoglienti o a realtà associative sensibili sui temi della solidarietà e della sussidiarietà e/o una pregressa esperienza di forme di accoglienza e/o affido familiare.

L'individuazione dei possibili beneficiari è avvenuta attraverso il coinvolgimento delle équipe di tutte le sedi dello SPRAR di Milano. Gli elementi presi in considerazione per la presentazione delle candidature al progetto sono stati: conoscenza livello A1/A2 lingua italiana; misure per l'integrazione già avviate; disponibilità/curiosità nei confronti di nuove relazioni; assenza di gravi vulnerabilità; sussistenza di un percorso positivo d'inserimento nel centro (buona relazione con l'équipe, rispetto delle regole e degli altri ospiti); assenza di progettualità alternative già strutturate; data prevista termine di accoglienza nello SPRAR. 
di neomaggiorenni maschi (figura 1 e 2). I beneficiari sono originari di oltre 15 Paesi (tabella 1) e sono stati accolti all'interno di 22 nuclei familiari. La discrepanza numerica tra ospiti e famiglie ospitanti si deve sostanzialmente a due fattori: alcune famiglie hanno deciso di ripetere l'esperienza, confermando la positività - sia per gli ospiti che per loro stessi - di quanto vissuto nei mesi di accoglienza; c'è stata inoltre una famiglia che ha ospitato presso la propria abitazione un nucleo familiare composto da tre persone.

Figura 1 - Fasce d'età dei beneficiari accolti dal 2016 al 2019

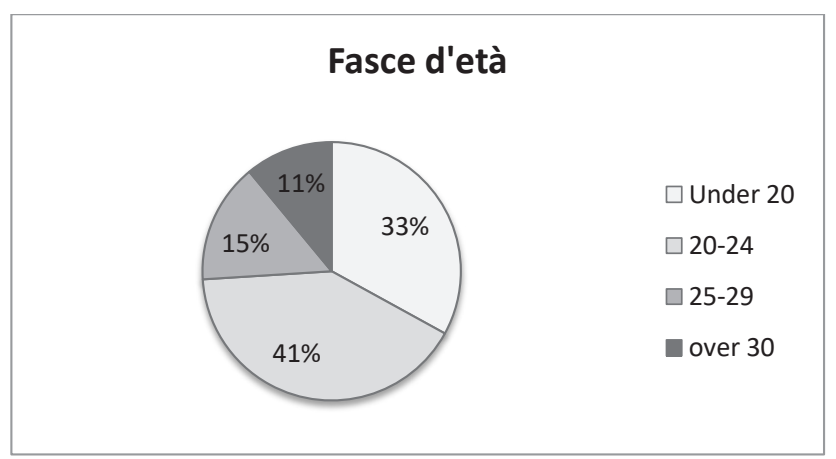

Figura 2 - Genere dei beneficiari accolti dal 2016 al 2019

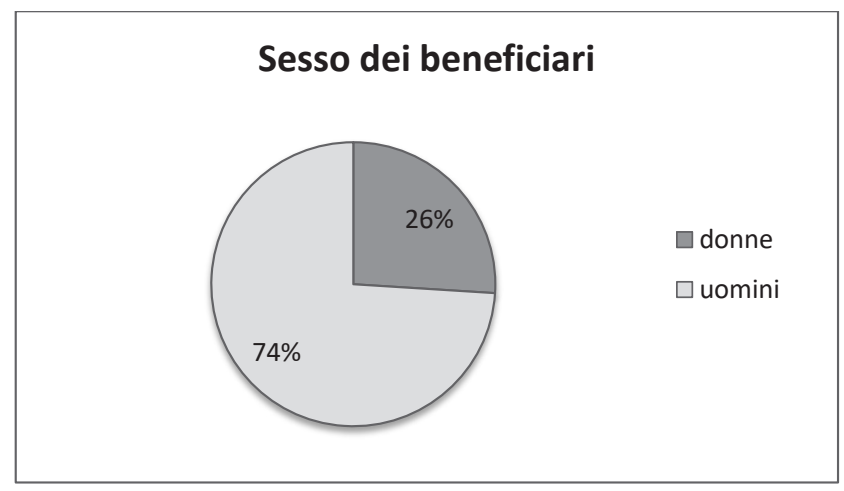

Tabella 1 - Nazionalità dei beneficiari accolti dal 2016 al 2019

\begin{tabular}{l|c}
\hline Nazionalità rappresentate & N. \\
\hline Afghanistan & 1 \\
\hline Burkina Faso & 1 \\
\hline Camerun & 1 \\
\hline Costa d'Avorio & 3 \\
\hline
\end{tabular}




\begin{tabular}{l|l}
\hline Etiopia & 2 \\
\hline Gambia & 2 \\
\hline Ghana & 1 \\
\hline Guinea Conakry & 1 \\
\hline Mali & 3 \\
\hline Nigeria & 1 \\
\hline Pakistan & 1 \\
\hline Senegal & 4 \\
\hline Somalia & 4 \\
\hline Sudan & 1 \\
\hline Uganda & 1 \\
\hline
\end{tabular}

Apparentemente questi piccoli numeri (27 accoglienze in quattro anni) stridono se confrontati con il numero di rifugiati e richiedenti asilo residenti presso i centri (il progetto SPRAR/SIPROIMI a Milano ogni anno accoglie circa 750 persone), tuttavia bisogna tener presente non solo che ad oggi si tratta di un progetto considerato, dallo stesso Servizio Centrale, ancora in fase sperimentale ma anche che in parte è proprio questa sua dimensione ridotta a consentire la cura particolareggiata di ogni singolo percorso. Si ipotizza quindi che l'accoglienza in famiglia, pur essendo una formula non utilizzabile su larga scala, rappresenti - sulla base della pur breve osservazione - un dispositivo dal valore aggiunto per diverse ragioni:

- offre alla persona accolta l'opportunità di una maggiore attenzione, focalizzata non solo sui macro-problemi (trovare casa e lavoro) ma anche sui dettagli e sugli stati d'animo di cui è intessuta la quotidianità. Tale attenzione può agevolare l'emersione delle esigenze personali più profonde ed autentiche, base per riuscire a rielaborare e/o definire il proprio progetto migratorio;

- permette, alle persone coinvolte e alle reti di relazioni loro più prossime, di incontrare e conoscere in modo non stereotipato sia le differenze culturali reciproche, sia i punti d'incontro comuni, premessa fondamentale per favorire reali e bidirezionali processi di integrazione;

- favorisce l'ampliamento e il potenziamento delle reti informali, a beneficio sia degli ospiti sia delle famiglie accoglienti;

- presenta, se ben restituita e comunicata, una dimensione di promozione culturale nei confronti dell'intera comunità locale.

\section{II Progetto e le sue fasi}

Le varie fasi progettuali vengono seguite da un'équipe multidisciplinare composta da professionisti sia dell'ente locale sia dell'ente gestore. L'équipe di riferimento del progetto è attualmente costituita da un assistente sociale del Comune di Milano, un coordinatore, un educatore e uno psicologo 
dell'ente gestore. La stretta collaborazione tra queste due realtà e tra differenti professionalità, arricchita dai continui rimandi provenienti dalle famiglie e dagli ospiti stessi, ha dato vita ad una circolarità virtuosa, producendo un continuo confronto tra le aree tecnico-professionali degli operatori coinvolti e le aree del "sapere esperienziale" vissuto dagli stessi protagonisti (Raineri, 2011).

\subsection{Selezione di famiglie e ospiti, abbinamento e conoscenza}

Per dare avvio alle singole accoglienze, l'équipe di progetto conosce da un lato le famiglie che si candidano, dall'altro i possibili beneficiari che vengono individuati e segnalati dai colleghi delle équipe dei centri di accoglienza ${ }^{4}$. Selezionati i candidati, la stessa équipe procede poi nell'individuare i possibili abbinamenti. Nella definizione dell'abbinamento vengono tenuti in considerazione differenti aspetti, tra cui anche le preferenze espresse da famiglie e ospiti durante i colloqui conoscitivi. Le richieste fin qui portate hanno riguardato prevalentemente, nel caso delle famiglie, il genere dell'ospite da accogliere e, nel caso dei futuri ospiti, la presenza o meno di animali domestici. I possibili abbinamenti vengono poi presentati ai diretti interessati e verificati nel corso della fase di reciproca conoscenza.

A tale scopo viene organizzato un primo incontro in uno spazio neutro alla presenza del beneficiario, di uno o più membri della famiglia, dell'operatore del progetto di accoglienza e dell'operatore di riferimento del Centro SPRAR/ SIPROIMI di provenienza. Successivamente viene fissato un incontro presso il domicilio della famiglia, in modo che il futuro beneficiario possa cominciare a conoscere anche gli spazi che lo ospiteranno. Infine, presso il Centro di accoglienza, si svolge il terzo incontro che coincide con il momento del trasferimento dell'ospite. Si è ritenuto opportuno infatti dare anche alle famiglie la possibilità di visitare i Centri ed incontrare gli operatori che vi lavorano per favorire la conoscenza dell'approccio di intervento dello SPRAR/SIPROIMI e facilitare così un confronto aperto sui modelli socio-educativi di riferimento. Rispetto all'organizzazione relativa di questa fase, si è assicurata comunque massima flessibilità, consentendo alle famiglie e agli ospiti di gestire, in accordo con l'operatore di riferimento, gli incontri successivi al primo, variandone il numero o diversificandone l'impostazione.

Per siglare la corresponsabilità di tutti gli attori coinvolti, l'équipe ha predisposto inoltre uno strumento metodologico denominato Accordo per l'attuazione del progetto "Rifugiato in Famiglia". L'accordo viene sottoscritto

\footnotetext{
${ }_{4}$ Il progetto si è dotato di strumenti funzionali alla gestione e allo sviluppo di ciascuna fase progettuale: scheda candidatura beneficiario, scheda candidatura famiglia, PEI (Progetto Educativo Individualizzato), Patto di accoglienza (sottoscritto da Comune di Milano, Cooperativa Farsi Prossimo, beneficiario, famiglia).
} 
presso gli uffici comunali, nei giorni immediatamente precedenti l'avvio dell'accoglienza.

\subsection{Avvio e supporto alla convivenza}

Iniziata la convivenza, I'educatore è la figura professionale più prossima alle famiglie e ai beneficiari. L'educatore svolge periodici colloqui di monitoraggio sia con gli ospiti sia con le famiglie, garantendo un supporto fondamentale all'esperienza in atto. Pochi giorni dopo il trasferimento dell'ospite in famiglia viene organizzato un incontro presso la casa, con l'educatore e lo psicologo del progetto, dove è possibile condividere un primo momento di osservazione e confronto rispetto alle dinamiche che cominciano ad instaurarsi all'interno delle mura domestiche. Nel corso dei sei mesi l'intera équipe accompagna I'accoglienza focalizzandosi su alcuni aspetti fondamentali quali: dinamiche relazionali, supporto alla famiglia, percorso individuale dell'ospite e accompagnamento all'autonomia e alla successiva uscita dal progetto.

All'accompagnamento delle singole esperienze si è deciso inoltre di affiancare un lavoro di gruppo, che permetta alle famiglie -impegnate nell'accoglienza simultaneamente- di confrontarsi tra loro, creando così I'opportunità di condividere temi e problematiche ricorrenti. Analogamente anche ai beneficiari vengono proposti dei momenti di gruppo per raccontarsi e conoscere le esperienze degli altri, con l'obiettivo di aumentare la loro conoscenza delle famiglie, quindi della società che li sta ospitando e nella quale stanno iniziando a ri-progettare il loro futuro.

\subsection{La conclusione dell'esperienza di accoglienza in famiglia}

Se nei primi mesi di accoglienza il focus si concentra maggiormente sul costituirsi di legami positivi, in un secondo tempo l'attenzione è rivolta maggiormente all'ospite e al suo percorso verso l'autonomia. Una particolare cura viene dedicata alla fase finale che assume, nei vissuti dei protagonisti, una rilevanza e un significato sempre crescenti. Gli operatori di riferimento hanno il compito di accompagnare in questa fase su un piano pratico ma anche emotivo l'ospite e la famiglia.

Nel concreto, I'educatore sostiene I'ospite nella ricerca di possibili soluzioni abitative economicamente sostenibili, coerenti con la capacità di spesa e di vita in autonomia dell'ospite (non è detto infatti che tutti gli ospiti alla fine dell'accoglienza abbiano raggiunto una completa stabilità professionale e una conseguente autonomia economica). In alcuni casi accade che sia la famiglia stessa a reperire risorse abitative per il proprio ospite.

A tutto ciò si affianca una presa in carico da parte dell'équipe dei vissuti emotivi collegati a questa fase di accoglienza. Come spesso capita nei momenti 
di separazione, i vissuti prodotti hanno una duplice natura: da un lato aprono a una possibilità di crescita, dall'altro richiedono un'elaborazione della perdita.

I beneficiari in alcuni momenti vivono l'esperienza di un passaggio all'autonomia finalmente compiuto, in altri si riattivano paure e bisogni di accudimento a causa dei quali si rischia di non sentirsi mai sufficientemente pronti. Al di là del sostegno dell'équipe, un ruolo fondamentale viene giocato dalla famiglia stessa che è chiamata a produrre la giusta spinta all'uscita evitando che ciò si traduca in una frattura del legame o in un'espulsione. Per questo motivo anche la famiglia viene supportata attraverso uno sguardo dedicato e la costruzione di occasioni di rielaborazione.

Conclusasi la convivenza la prosecuzione del rapporto tra famiglia accogliente e ospite riveste un valore aggiunto che non contraddistingue tutte le esperienze, non va data dunque per scontata in modo automatico e può assumere forme e modalità differenti. Alcuni ospiti interpretano la raggiunta autonomia con una presa di distanza da quei luoghi e quelle relazioni che li hanno visti in posizione di dipendenza; altri invece mantengono un rapporto costante, alle volte acquisendo le abitudini italiane per cui - per esempio - ci si ritrova per il pranzo domenicale. In entrambi i casi però, il più delle volte, la famiglia resta un punto di riferimento importante, una porta a cui bussare in caso di necessità o per il desiderio di mostrare e condividere i progressi conquistati.

\section{Nella quotidianita' delle accoglienze}

L'ospitalità in famiglia, sebbene sia uno strumento di accoglienza inserito nella progettazione istituzionale, a differenza di altre forme di accoglienza offerte dal sistema SIPROIMI, si gioca su un terreno di natura diversa. II beneficiario infatti è inserito in un contesto radicalmente nuovo, che lo chiama in causa in prima persona: non più uno fra i tanti fruitori di un servizio ma ospite nel senso più proprio del termine; accolto in un luogo che di per sé è impregnato di una nuova cultura da conoscere, in una potenzialità di relazioni da costruire; il tutto giocato all'interno della vita quotidiana, in quelle che si definiscono aree interstiziali della quotidianità (Gasparini, 2004). In famiglia anche solo la condivisione di alcune regole di convivenza prende un sapore nuovo, non più solo norme da seguire, pena il richiamo e/o la sanzione, ma abitudini - per l'appunto - familiari, da imparare e rispettare per prendersi cura del legame reciproco. In un terreno della vita quotidiana come quello domestico può capitare allora che emergano dimensioni latenti che non hanno spazio per emergere in altri contesti di accoglienza; ciò diviene da un lato occasione preziosa per accogliere e trattare elementi nuovi dall'altro, il sorgere di temi inaspettati, aumenta la complessità dell'esperienza stessa. Di 
seguito si proverà a presentare alcune di queste variabili, dando spazio anche alla voce di coloro i quali in questi anni sono stati protagonisti del progetto ${ }^{5}$.

\subsection{Lo spazio della casa: ritrovata privacy e nuovi spazi di condivisione}

Il luogo dove l'esperienza di "Rifugiato in famiglia" prende corpo è quello della casa, che assume in sé diverse valenze; ciò è ancora più rilevante se si considera che i rifugiati provengono da un'esperienza di abbandono forzato della propria terra e della propria casa e vivono nella nazione che li ospita un lungo periodo di interregno prima di arrivare nuovamente ad abitare un luogo che possano chiamare casa. La casa per queste persone inoltre può aver perso quella dimensione di luogo sicuro che invece dovrebbe esserle proprio, facendo così venire a mancare anche quella tranquillità e libertà che consente alle persone di pensare a loro stessi e al proprio futuro.

Tutti gli ospiti accolti in famiglia provengono da un periodo, anche molto lungo, di quotidianità trascorsa in uno o più centri di accoglienza e tornare ad abitare lo spazio di una casa, per quanto possa coincidere con i loro desideri, è un passaggio delicato; tanto più perché si tratta di abitare la casa di qualcun altro, assumendo così un nuovo abito da ospite e non ancora una reale autonomia. A questa complessità si aggiunge inoltre il non irrilevante dettaglio della differenza culturale nella quale si andrà a sviluppare la convivenza.

Per tali motivi questa progettualità offerta, ma mai imposta, ai possibili beneficiari non sempre viene accolta positivamente. Alcuni ospiti possono vedere in questa esperienza un'occasione di crescita e un'opportunità, altri invece ritengono che abbia un costo in termini relazionali e di autonomia difficile da sostenere.

D'altro canto decidere di accogliere è un'esperienza a cui solo alcune famiglie si aprono: ripensare alla propria privacy e libertà in funzione di un estraneo, con cui condividere il proprio spazio domestico, non è una scelta che si può assumere con leggerezza.

La cosa bella è stata la naturalità con cui è entrato a far parte della nostra famiglia, della nostra realtà familiare. Con molta discrezione. (familiare)

La discrezione dunque come skill che facilita I'avvio della convivenza mostra come la qualità dell'esperienza del progetto "Rifugiato in famiglia" si costruisca anche attraverso i piccoli gesti che definiscono la quotidianità. Proprio per questo motivo un'attenzione particolare deve essere dedicata alla gestione di spazi, tempi e regole della casa. Entrare infatti in una casa di persone

\footnotetext{
A tale scopo si è deciso di utilizzare alcuni frammenti di interviste presenti nel lavoro di ricerca di dottorato di una delle autrici di questo articolo (Tagliabue, 2017). La ricerca ha approfondito il tema dell'accoglienza in famiglia ed in particolar modo il progetto implementato dal Comune di Milano.
} 
sconosciute non è come abitare in un centro collettivo che apparentemente non appartiene a nessuno. Giorno dopo giorno ospitanti e ospitati sono chiamati a costruire insieme un nuovo equilibrio che possa rispettare gli spazi di condivisione e di autonomia di ciascuno. Contemporaneamente nello spazio di libertà della casa, a differenza dei centri collettivi in cui la quotidianità è regolamentata da un altro istituzionale, si può recuperare anche una dimensione di maggiore intimità che facilita il pensare a se stessi e alla propria progettualità.

È un'altra vita nella famiglia di quando vai al centro di accoglienza. Tranquillo, puoi decidere, puoi pensare e poi in centro d'accoglienza non puoi pensare, non puoi decidere niente, sei sempre nervoso, sempre hai problemi mentali perché ci sono cinquanta persone che vivono insieme urlano, parlano, litigano, no? (ospite)

Il recupero della propria individualità e il conseguente desiderio di autonomia richiede che all'esperienza di intimità si affianchi tuttavia il rispetto del territorio indipendente di ciascuno (Komter, Van Leer, 2012), solo in questo modo la grande vicinanza fisica e affettiva che può aver luogo in una casa non corre il rischio di essere percepita come un'invasione. Diversamente può accadere che le differenze e le distanze si acutizzino rendendo faticosa la convivenza. Quando ciò si verifica e si allenta la condivisione, la distanza che si viene a creare pesa su tutti i soggetti coinvolti: da un lato le famiglie ospitanti non percepiscono una risposta partecipata a ciò che hanno offerto in termini di vicinanza, dall'altro gli ospiti non si sentono riconosciuti nella propria autonomia.

I: Ho parlato con amici di questa mia preoccupazione di entrare in famiglia, solo una: si dice in inglese privacy di famiglia, la vostra privacy, non la mia. Per esempio quando posso stare con famiglia o quando no, qual è il tempo di vostra privacy.

[...]

I: La mia privacy sempre, lui mai disturba. Non ho visto in sette mesi [ospitante] entrare nella mia stanza.

R: La gestivi solo tu?

I: Sempre solo io, anche mio bagno. Quando lui vuole parlare con me, mi chiama sempre, ma mai entra in mia stanza, mai.

R: Ve lo siete detti...

I: No, è stato così, non comunicato, mai, come si dice in inglese... Understanding! (ospite)

\subsection{Legami che consentono di proiettarsi in un futuro autonomo}

Nell'accoglienza in famiglia i rapporti che si vengono a creare tra i soggetti coinvolti possono assumere contorni e legami differenti. Ciò che si verifica sembra assumere caratteristiche simili, seppure con le dovute differenze trattandosi di adulti, a quello che accade nelle esperienze di affido dei minori stranieri non accompagnati. Prendendo dunque in prestito la categorizzazione proposta da Sirriyeh (2013), che nei suoi studi ha approfondito quanto avviene 
nella relazione tra minore straniero non accompagnato e famiglia accogliente, si possono evidenziare tre tipologie di legami che si producono: like-family (come di famiglia); guest (ospite); lodgers (inquilino). Tali categorie sembrano poter essere rappresentative della qualità e tipologia dei legami anche del progetto "Rifugiato in Famiglia".

Perché c'è differenza: a [nome Centro] con educatori e assistente sociale loro lavoravano io ero come un ospite. Niente altre, si dice in inglese, relations. Ma per esempio io vivo con [nome ospitante], in sei mesi lui diventa mio fratello perché io parlo con lui di qual è mio problema, lui mi dà consiglio [...] così... I get suggestions from him, so... è così. (ospite)

Nella prima tipologia di legame proposto da Sirriyeh le relazioni sono contraddistinte dalla costruzione di legami forti che sembrano avvicinarsi alle relazioni familiari (like-family). In queste situazioni può accadere che le persone coinvolte nell'esperienza descrivano il rapporto con termini come "fratello", "figlio", "mamma".

Nella seconda tipologia presentata dalla studiosa, la relazione tra ospite (guest) e famiglia viene a delinearsi come un rapporto di rispetto vicendevole e fiducia, anche di affetto, in cui le relazioni tuttavia non si descrivono in termini familiari. Si passa il tempo insieme e si decide di partecipare con coinvolgimento alla vita familiare senza sentirsene totalmente parte. Si tratta di una dinamica piuttosto frequente che risponde da un lato alla durata relativamente breve del progetto, dall'altro sembra essere una sorta di meccanismo di difesa: la famiglia che accoglie rischia di essere vista solo come l'ennesimo passaggio, prima del raggiungimento della tanto desiderata autonomia.

Direi che ad un certo punto uno deve uscire dalla sua zona di comfort, magari tu ci sei abituato vivendo in un centro d'accoglienza... non so come dire, ti trasforma no? In qualche modo, magari lì non è che sei cattivo o sei gentile, ma lì ti trasforma perché ogni ambiente ha le sue realtà. Quindi vivendo in un centro d'accoglienza diventi un $\mathrm{po}^{\prime} . .$. non so come dire, arrivano sempre nuove persone, vanno via altre con cui sei abituato... quindi diventi un po' superficiale nel rapporto con la gente che vedi, che viene e che va, che viene e che va. [...] Quindi diventi un po'... metti un po' di barriera, rimani un po' nel tuo angolino, ti fai un po' una tua zona di comfort. [...] A un certo punto ho detto "no, adesso esco da questa dinamica, da questo modo di fare, perché adesso vivo con una famiglia e la famiglia è molto importante", no? Una famiglia! (ospite)

Quando i beneficiari del progetto per le più svariate ragioni non riescono a lasciare la suddetta zona di comfort tendendo a vivere molto più lo spazio della stanza che quello della casa, si verificano delle situazioni di ospitalità contraddistinte da un rapporto di affittuario/inquilino (lodgers). In questi casi emerge una scarsa condivisione degli spazi e una certa distanza tra i diversi soggetti, che rischia di affaticare l'esperienza in corso per tutte le persone coinvolte; ciò può pregiudicare anche la prosecuzione del rapporto successivamente al periodo di accoglienza. L'instaurarsi di questo ultimo tipo di 
relazione tuttavia non può essere letto automaticamente come un insuccesso dell'accoglienza in famiglia. Questa esperienza infatti può rispondere, come già sottolineato, ad esigenze diverse: per qualcuno è l'occasione per ritrovare il calore della dimensione familiare, per altri invece è un modo per uscire dalla dimensione del centro collettivo per una più veloce riconquista dell'autonomia.

Comunque è andato bene. [...] Perché anche quando ho finito qua [nome ospitante] ha detto... Ogni sabato/domenica vieni a mangiare... Puoi venire a fare quello che vuoi, qua è casa tua. E quindi così, per me è andata molto bene. (ospite)

Speriamo comunque bene tutto sommato... poi ha noi, poi ha la rete familiare nostra. Quindi non è che è proprio completamente sperso. Per cui se avesse bisogno di qualcosa... comunque i legami si sono creati... (familiare)

Il lavoro degli operatori professionali del progetto spesso è quindi proprio quello di aiutare le persone coinvolte a rileggere quanto accade tra le mura domestiche, nel tentativo di attribuire un nuovo significato alle complessità che possono segnare i differenti modi di stare nel legame e disvelarne le motivazioni sottostanti.

A prescindere dalla forma della convivenza si è osservato che la creazione di legami, che possano durare nel tempo, ha spesso permesso agli ospiti di sentirsi più forti. Poter contare su dei legami nel nuovo territorio, che rinforzano le autonomie acquisite e divengono una rete di supporto nel caso di necessità, permette loro di pensarsi con più serenità e sicurezza in un futuro autonomo.

\section{Conclusioni: I’accoglienza in famiglia come forma di cura della polis}

È come... per me è stata una esperienza molto importante in quel momento del mio percorso dell'accoglienza. In quel momento lì è stata come una mano che è uscita da qualche parte a salvarmi, ecco. (ospite)

Questa frase ci propone un'immagine che adombra contemporaneamente la forza e il nodo critico delle esperienze di accoglienza. Mani che salvano, Paesi che accolgono e famiglie che aprono la propria porta ci parlano indubbiamente di una capacità dell'essere umano di accogliere lo straniero, disposizione dell'animo imprescindibile eppure non sufficiente affinché si dia origine a dei reali percorsi di integrazione. Come sostiene Derrida, I'accoglienza prende sempre le mosse a partire da una posizione di disequilibrio:

La questione dell'ospitalità comincia da qui: dobbiamo chiedere allo straniero di capirci, di parlare la nostra lingua, nell'accezione più vasta del termine, in ogni possibile accezione, prima di accoglierlo in casa nostra, e anzi quale condizione necessaria? Se invece parlasse già la nostra lingua, con tutto ciò che la cosa implica, se condividessimo già tutto ciò che insieme alla lingua si condivide, lo straniero sarebbe ancora uno straniero e potremmo al suo riguardo parlare di asilo o di ospitalità?. (Derrida, Dufourmantelle, 2000, p. 46-47) 
Alla difficoltà di rinunciare alla propria lingua madre per acquisire quella del luogo, condizione tipica del turista in un Paese straniero, per i richiedenti asilo e i rifugiati politici si aggiunge inevitabilmente anche la drammaticità di non avere una casa, un lavoro e una stabilità sociale ed economica. In una cornice - così palesemente di svantaggio - accogliere diventa il primo passo necessario; tuttavia affinché vi siano dei reali percorsi di integrazione e non solo un processo di assimilazione (Berry 1997, 1998) altrettanta rilevanza hanno tutti quei passi successivi che vanno nella direzione di ridurre al minimo il gap fin qui descritto.

Si ritiene infatti che non vi possa essere ospitalità ed integrazione se non in una esperienza che ridia protagonismo all'ospite stesso, favorendo il recupero di una dimensione identitaria e progettuale che permetta di rinsaldare quel senso di appartenenza e di cura, che nasce dal riconoscere di essere trattati come soggetti attivi e capaci di operare delle scelte (Tagliabue, 2019).

Come evidenziato nei precedenti paragrafi, tutto ciò sembra aver luogo più velocemente tra le mura domestiche più di quanto non accada nei grandi centri di accoglienza e nel calore della relazione affettiva in alternativa a quella di stampo professionale. Lo spazio della casa favorisce la possibilità di trovare tempo per stare e per pensare, quasi indipendentemente dal raggiungimento di alcuni obiettivi concreti pure indispensabili - quali il lavoro e l'alloggio. La dimensione domestica sembra quindi sostenere, oltre alla riconquista dell'autonomia, un'inedita percezione di sé che integri identità e nuove esperienze di appartenenza.

Attraverso il periodo di convivenza, inoltre, gli ospiti (termine che etimologicamente indica sia chi ospita sia chi è ospitato) possono vivere insieme momenti di attesa, di viaggio, di gioco, di spaesamento, di sorpresa, di silenzio (Gasparini, 2004). Il concetto di integrazione viene in questo modo ad ampliarsi, comprendendo quell'aspetto bidirezionale che è caratteristica sostanziale dei processi di integrazione e che influenza le differenti dimensioni proposte dagli studiosi Ager e Strang $(2008)^{6}$ a cui si farà riferimento.

Tuttavia è importante richiamare anche alcune criticità dell'accoglienza in famiglia, che riveste indubbiamente un valore aggiunto ma in nessun caso - si

6 I due studiosi hanno costruito un framework che evidenzia in modo sintetico le dimensioni dell'integrazione. La mappa ha la forma di una piramide rovesciata (figura 3) nel cui punto più alto sono presenti i markers and means, ossia le dimensioni più riconosciute come esiti di integrazione nella nuova società (come per esempio il lavoro e la casa). Nell'area posizionata sulla parte più bassa, alla punta della piramide, sono evidenziate le dimensioni che gli autori definiscono foundation (fondamenta) rappresentate dai diritti e dalla cittadinanza. Nell'analizzare le aree centrali della piramide, Ager e Strang riflettono su quali siano quegli elementi che permettono di costruire "tessuto connettivo" tra i diritti che rappresentano le fondamenta dell'integrazione e le dimensioni espresse dai markers and means. I due autori ci parlano di social connection and facilitators come rilevanti nel creare questo tessuto connettivo. 
ritiene - possa essere considerata alternativa all'accoglienza di tipo istituzionale. L'ospitalità in famiglia infatti consente di rispondere alle esigenze di una minima parte dei rifugiati politici e, quand'anche fosse utopicamente possibile, non si ritiene auspicabile che si sostituisca al prezioso lavoro svolto nei centri collettivi, evidentemente pensati come luoghi contraddistinti da professionalità ed accoglienza.

Abbiamo visto, anche grazie alle parole di un ospite, come scegliere di co-abitare in una casa richieda da entrambe le parti la momentanea rinuncia alla propria zona di comfort, esperienza mai scontata. A ciò si aggiungano la complessità e la drammaticità di vissuti e traumi, che lo straniero in fuga porta inevitabilmente con sé. La mancanza dei documenti, la possibile presenza di patologie organiche, quando non di ferite, i disturbi di tipo psicologico e/o psichiatrico, il pesante bagaglio di dolore, il lutto per quanto lasciato alle proprie spalle, tratteggiano un quadro spesso di fragilità che non può non chiamare in causa anche le competenze multidisciplinari, proprie di una buona accoglienza istituzionale.

In questa ottica si può affermare che il progetto "Rifugiato in Famiglia", qui presentato, si collochi in una terra di mezzo tra le due tipologie di accoglienza. In Italia il Servizio Centrale chiede infatti che lo strumento dell'accoglienza in famiglia venga inserito non solo all'interno di una progettazione locale, che ne valuti la fattibilità e la modalità di realizzazione, ma che si costruisca - anche in termini di garanzia - una collaborazione, un circuito virtuoso quadrangolare tra i diversi attori coinvolti: lo SPRAR/SIPROIMI territoriale, attraverso l'équipe di riferimento dell'ente gestore; il Comune, mediante i suoi servizi sociali; le famiglie, per l'effettiva accoglienza; i diretti interessati, titolari di protezione internazionale. La definizione stessa di "circuito virtuoso quadrangolare" mette in evidenza il co-protagonismo di tutti i soggetti a vario titolo coinvolti, compresi quei migranti di cui si è cercato di tratteggiare l'iniziale posizione di diseguaglianza. Tale tentativo di riallineamento delle posizioni non può aver luogo che a partire dalla disponibilità degli altri tre protagonisti (Comune, ente gestore e famiglia) a rinunciare ad un po' del proprio potere in favore della persona ospitata. In questo percorso di accoglienza si evince cioè una scelta di cessione del potere che viene suddiviso tra i vari soggetti in gioco coinvolgendo anche i diretti interessati, come se in questo processo le politiche e le pratiche potessero essere tenute in comunicazione attraverso la suddetta bidirezionalità che influenza i diversi attori (Tagliabue, 2017). II soggetto beneficiario dell'accoglienza diviene attore e responsabile delle proprie scelte, consapevole che queste avranno delle conseguenze anche su chi gli sta intorno. Le famiglie che accolgono accettano di aprire la porta di casa non solo agli ospiti ma anche a quegli operatori che, a vario titolo, accompagneranno l'esperienza. Contemporaneamente l'operatore stesso, 
stante che in genere nella nostra cultura tecnologica è il professionista che tende ad avere maggior potere d'azione, entra in un processo di cessione del proprio potere (Folgheraiter, 1998), condividendolo con le altre figure della rete in cui è intessuta l'accoglienza.

Compito dell'operatore diviene dunque quello di sovra-intendere il processo di aiuto in modo da facilitare il bilanciamento dei vari apporti. L'operatore, rinunciando a parte del proprio controllo sul processo, compie un passo indietro e si dedica in particolar modo alla cura di quelle dinamiche relazionali che consentono un inedito accostarsi all'altro. Conoscere lo straniero, nello svolgimento della vita quotidiana e nella vicinanza, consente di ri-conoscerlo come soggetto di diritto, capace di scegliere e di essere accompagnato, ma non diretto, nella ri-conquista di una propria autonomia. Utilizzando le riflessioni proposte dagli studiosi Ager e Strang (2008) (figura 4) in merito alle dimensioni dell'integrazione, durante questi primi quattro anni di implementazione del progetto si è potuto osservare infatti come questo tipo di accoglienza in famiglia sia intervenuta non solo sulle dimensioni dell'integrazione che richiamano le interazioni sociali (social connection) - nello specifico sui legami con la popolazione locale (social bridges), favorendo lo sviluppo di conoscenze culturali e linguistiche (language and cultural knowledge) -, ma abbia anche sostenuto gli ospiti nel sentirsi riconosciuti nei propri diritti umani e di cittadinanza (rights and citizenship). Come testimoniato dalle interviste, tale modalità ha contribuito ad accrescere la loro percezione e l'esperienza diretta di sicurezza e stabilità (safety and stability), agendo sulle dimensioni dell'integrazione che toccano le fondamenta e i meccanismi di facilitazione di questi processi (foundation and facilitators) (Ager, Strang, 2004).

Figura 3 - Framework concettuale delle dimensioni dell'integrazione

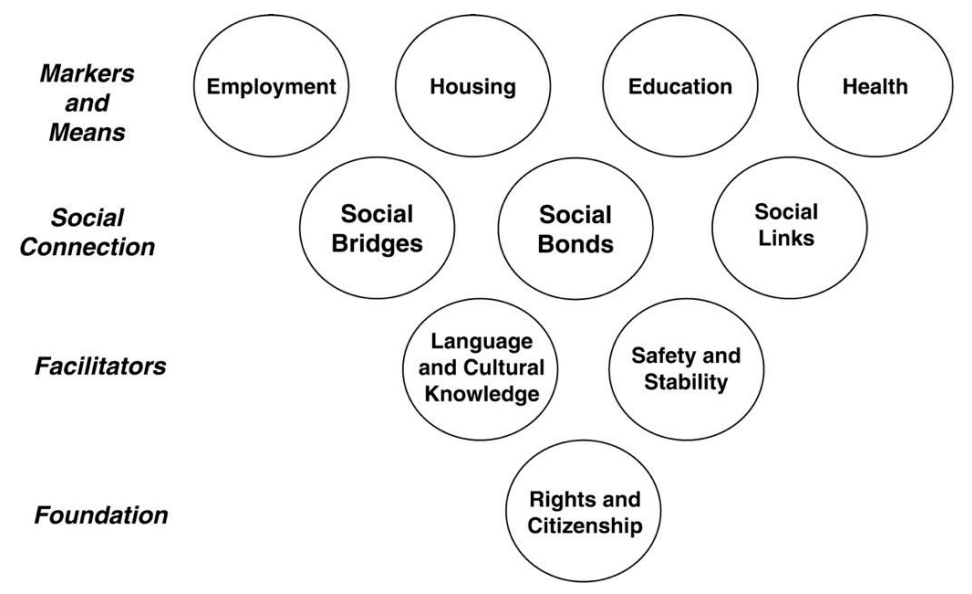


È possibile quindi affermare che questo meccanismo di rinuncia alla zona di comfort, al controllo e all'uso di un certo potere, abbia prodotto di contro per i beneficiari un surplus in termini di "diritto di avere diritti" (Arendt, 2009).

Ma qual è il vantaggio degli altri attori coinvolti, nel rinunciare ad un pezzetto del proprio protagonismo in favore di quello altrui? Si tratta di un quesito importante che tuttavia si è delineato con maggior chiarezza solo di recente, con il consolidarsi dell'esperienza. Una questione ancora aperta per la quale, per il momento, si è solamente abbozzata una risposta parziale. Le famiglie aprendosi all'accoglienza e alla contaminazione con lo straniero, possono cogliere l'occasione di sperimentare "forme di cittadinanza radicate nei contesti di residenza, secondo la via tracciata dalle numerose esperienze di cittadinanza 'agita' e partecipata, protagoniste di una forma 'generativa' di cittadinanza" (Zanfrini, 2016, p. 67). Inoltre si ipotizza che l'istituzione pubblica, in collaborazione con il privato sociale, si apra ad una pratica che, in sinergia alle pur necessarie azioni di supporto assistenziale, includa una cura della polis che si concretizza attraverso interventi di sviluppo della comunità. In tal modo emerge che ad essere sostenuta

È un'idea di cittadinanza che chiama in causa la capacità autoriflessiva della società, che si nutre del confronto di esperienze e prospettive diverse, ma che al tempo stesso lega i cittadini, cioè le persone che convivono all'interno della polis, attraverso il loro comune impegno per la costruzione della convivenza e del bene comune. (Zanfrini, 2016, p. 67)

\section{Bibliografia}

AGER Alastair; STRANG Alison. Indicators of Integration: Final Report. Home Office Development and Practice Report 28. London, 2004. Reperibile online: < https:// webarchive.nationalarchives.gov.uk/20110218141321/http://rds.homeoffice. gov.uk/rds/pdfs04/dpr28.pdf >. Visitato: novembre 2019.

AGER, Alastair; STRANG, Alison. Understanding Integration: A Conceptual Framework. Journal of Refugee Studies, v. 21, n. 2, p. 166-191, 2008.

ARENDT, Hannah. Le origini del totalitarismo. Torino: Einaudi, 2009.

BERRY, John. Widdup. Acculturation and health. In: KAZARIAN Shahe S.; EVANS David R. (eds.). Cultural clinical psychology: Theory, research and practice. Oxford: Oxford University Press, 1998, p. 39-57.

BERRY John Widdup. Immigration, acculturation, and adaptation. Applied Psychology: An International Review, v. 46, n. 1, p. 5-34, 1997.

DERRIDA, Jacques; DUFOURMANTELLE, Anne. Sull'ospitalità: le riflessioni di uno dei massimi filosofi contemporanei sulle società multietniche. Milano: Baldini\&Castoldi, 2000.

FOLGHERAITER, Fabio. Teoria e metodologia del servizio sociale: La prospettiva di rete. Milano: Franco Angeli, 1998. 
GASPARINI, Giovanni. Le piccole cose: interstizi e teoria della vita quotidiana. Milano: Guerini e Associati, 2004.

KOMTER, Aafke; LEER, M. Van. Hospitality as a gift relationship: Political refugees as guests in the private sphere. Hospitality \& Society, v. 2, n. 1, p. 7-23, 2012.

MINISTERO DELL'INTERNO. Associazione Nazionale Comuni italiani, Servizio centrale del sistema di protezione per richiedenti asilo e rifugiati. Manuale operativo per l'attivazione e la gestione di servizi di accoglienza integrata in favore di richiedenti e titolari di protezione internazionale e umanitaria. 2015. Reperibile online: <https://www.sprar.it/wp-content/uploads/2016/06/SPRAR__Manuale_ operativo_2015.pdf>. Visitato: novembre 2019.

RAINERI, Maria Luisa. II valore delle conoscenze esperienziali. In: DONATI, Pierpaolo; FOLGHERAITER, Fabio; RAINERI, Maria Luisa (eds.). La tutela dei minori: Nuovi scenari relazionali. Trento: Erickson, 2011, p. 87-101.

SIRRIYEH, Ala. Hosting strangers: hospitality and family practices in fostering unaccompanied refugee young people. Child \& Family Social Work, v. 18, p. 5-14, 2013.

TAGLIABUE, Claudia. Rifugiato in famiglia: Uno studio di caso su una nuova forma di accoglienza per richiedenti asilo e titolari di protezione internazionale e umanitari (Tesi di dottorato). Università Cattolica del Sacro Cuore di Milano, dottorato di ricerca in Sociologia, Organizzazioni e Culture, XXX Ciclo, S.S.D: SPS/07, a.a. 2016-2017.

TAGLIABUE, Claudia. Un rifugiato in famiglia. Lavoro sociale, v. 19, n. 2, p. 10-19, 2019.

ZANFRINI, Laura. Verso una nuova idea di cittadinanza. La sfida dei migranti per la società civile e per la Chiesa. La rivista del Clero Italiano, n. 1, p. 55-68, 2016. 